\title{
Continuous Finite-Time Output Regulation of Nonlinear Systems With Unmatched Time-Varying Disturbances
}

DOI:

10.1109/LCSYS.2017.2755363

\section{Document Version}

Accepted author manuscript

Link to publication record in Manchester Research Explorer

\section{Citation for published version (APA):}

Yang, J., Ding, Z., Li, S., \& Zhang, C. (2017). Continuous Finite-Time Output Regulation of Nonlinear Systems With Unmatched Time-Varying Disturbances. IEEE Control Systems Letters, 2(1).

https://doi.org/10.1109/LCSYS.2017.2755363

\section{Published in:}

IEEE Control Systems Letters

\section{Citing this paper}

Please note that where the full-text provided on Manchester Research Explorer is the Author Accepted Manuscript or Proof version this may differ from the final Published version. If citing, it is advised that you check and use the publisher's definitive version.

\section{General rights}

Copyright and moral rights for the publications made accessible in the Research Explorer are retained by the authors and/or other copyright owners and it is a condition of accessing publications that users recognise and abide by the legal requirements associated with these rights.

\section{Takedown policy}

If you believe that this document breaches copyright please refer to the University of Manchester's Takedown Procedures [http://man.ac.uk/04Y6Bo] or contact uml.scholarlycommunications@manchester.ac.uk providing relevant details, so we can investigate your claim.

\section{OPEN ACCESS}




\title{
Continuous Finite-Time Output Regulation of Nonlinear Systems With Unmatched Time-Varying Disturbances
}

\author{
Jun Yang, Zhengtao Ding, Shihua Li, and Chuanlin Zhang
}

\begin{abstract}
In this letter, a new continuous nonlinear controller ensuring finite-time output regulation is proposed for a class of nonlinear systems subject to unmatched time-varying disturbances. The controller is developed in a composite manner which combines invariant manifold principle, feedback domination and disturbance observation techniques. A key idea is to realize the finite-time estimation of system steady states by means of finite-time disturbance observers. A feedback domination approach with dynamic disturbance compensation in each step of virtual control design is then developed by using the tool of adding a power integrator. Finite-time stability of the closedloop system is established by means of Lyapunov theories, which shows that finite-time convergence of output tracking error is guaranteed even in the presence of time-varying disturbances subject to mismatching condition. The claimed performances of the proposed method are validated by a simulation example.
\end{abstract}

Index Terms-Nonlinear system, finite-time exact tracking, disturbance observer, time-varying disturbances, mismatching condition.

\section{INTRODUCTION}

The design of a nonlinear controller to ensure satisfactory tracking and disturbance rejection performance for disturbed nonlinear systems is one of the central design problems in nonlinear control theory [1]-[5]. One possible way to address this issue is to suppress the undesired effects of disturbances to a pre-described level via a relatively higher control energy, see nonlinear $H_{\infty}$ control [1], [2] and robust backstepping control [3] for examples. Those kinds of controllers could only achieve the goal of practical rather than exact stabilization/tracking.

Disturbance compensation by means of disturbance estimations has provided a feasible manner to achieve exact stabilization/tracking of nonlinear systems [5]-[9]. For lower-triangular nonlinear systems with unmatched time-varying disturbances under consideration, there are mainly two categories of control approaches focusing on addressing the exact tracking control problem, which are summarized as follows.

- Nonlinear Output Regulation [4], [5], [9], [10]: By utilizing internal model principle, output regulation provides a feasible way to address the exact tracking control problem of the systems under consideration. However, it is usually supposed that the external disturbances are governed by certain deterministic (if not exactly known)

J. Yang and S. Li are with School of Automation, Southeast University, Key Laboratory of Measurement and Control of CSE, Ministry of Education, Nanjing 210096, P.R. China (e-mails: j.yang84@ @eu.edu.cn and 1sh@seu.edu.cn).

Z. Ding is with School of Electrical and Electronic Engineering, University of Manchester, Sackville Street Building, Manchester M13 9PL, UK (e-mail: zhengtao.ding@manchester.ac.uk).

C. Zhang is with the College of Automation Engineering, Shanghai University of Electric Power, Shanghai 200090, China (e-mail: clzhangseu@gmail.com). exosystems [5]. When the external disturbances is not generated by deterministic exosystems or the exosystems are completely unknown, it is difficult (if not impossible) for the existing output regulation approaches to achieve exact tracking and disturbance rejection [9]. The reason is that no disturbance model information is available and hence it is unable to design an internal model in output regulation approaches to reconstruct the disturbance model.

- Backstepping+HOSM [13]-[18]: The combination of backstepping design and higher-order sliding mode (HOSM) techniques provides an alternative to exact tracking of nonlinear systems subject to general disturbances. Note that the two methods in [13], [14] only guarantee asymptotical exact tracking rather than finitetime exact tracking. The paper [15] proposed a finitetime exact tracking control approach, while the control law is discontinuous therein since the HOSM controller is taken. In [16]-[18], the finite-time exact tracking problem is addressed for linear dynamic systems subjec$\mathrm{t}$ to unmatched disturbances/uncertainties. Furthermore, "explosion of complexity" is generally caused by repeated calculations of virtual controllers for basic backstepping approaches.

It has been reported that the finite-time stable systems have two remarkable advantages: faster convergence speed as well as better disturbance rejection performance (see [19]-[23] and the references therein). Consequently, we aim to address the problem of finite-time exact tracking and disturbance rejection of a class of lower-triangular nonlinear systems subject to unmatched time-varying disturbances. In general, the mismatching condition means that the disturbances enter via different channels from those of the control inputs. By virtue of the concept of invariant manifolds in output regulation theory, the steady states of the disturbed system are solved and represented in terms of various derivatives of disturbance/reference signals. The finite-time estimation of the steady states is achieved by adequately exploiting the finite-time disturbance observers. Combining generalized disturbance cancelation in each step of the recursive design, a composite feedback domination approach is newly proposed by further exploiting the technique of adding a power integrator [19], [24]. It is shown by Lyapunov stability theory that the proposed method ensures the finite-time exact tracking control of nonlinear systems under unmatched time-varying disturbances. In addition, it is proved that all system states together with the control input reach their pre-calculated steady-state values accurately in finite time. Since "explosion 
of complexity" caused by repeated calculations of virtual controllers in basic backstepping approaches is avoided here, the presented methodology yields a concise control law for implementation. To the best knowledge of the authors, this is the first work on continuous finite-time exact tracking control (rather than asymptotical exact tracking control or discontinuous finite-time exact tracking control) of lower-triangular nonlinear systems with unmatched time-varying disturbances. Finally, the effectiveness of the proposed method is finally demonstrated by a numerical example.

\section{NotATIONS AND Some Useful Lemmas}

\section{A. Notations}

The following notations are provided for briefness of expressions.

- The symbols $\mathbb{Q}_{\text {odd }}^{+}$and $\mathbb{Q}_{\text {odd }}^{\geq 1}$ denote the set of ratio of two positive odd integers, and set of ratio that greater than 1 , of two positive odd integers, respectively. For integers $j$ and $k$ satisfying $0 \leq j \leq k$, let $\mathbb{N}_{j: k}:=\{j, j+1, \cdots, k\}$ be a set of nonnegative integers. The symbol $\mathcal{C}^{i}$ denotes the set of all differentiable functions whose first $i$ th time derivatives are continuous. The symbol $\mathcal{L}_{\infty}$ represents the set of all signals whose infinity-norms are bounded.

- For any $x_{i} \in \mathbb{R}$, denote a real vector $\vec{x}_{i}:=\left[x_{1}, \cdots, x_{i}\right]^{T}$. This definition will also be used for real vectors $\pi_{i}, \hat{\pi}_{i}$ and $\eta_{i}$. For any $w_{i} \in \mathbb{R}, r \in \mathbb{R}$, we define three real number sets as $\bar{w}_{i, j}:=\left\{w_{1}^{(j)}, w_{2}^{(j)}, \cdots, w_{i}^{(j)}\right\}$ (for $i \in \mathbb{N}_{1: n+1}$ and $\left.j \in \mathbb{N}_{0: i-2}\right), \mathbf{w}_{i}:=\left\{\bar{w}_{i-1,0}, \bar{w}_{i-2,1}, \cdots, \bar{w}_{1, i-2}\right\}$, and $\mathbf{r}_{i}:=\left\{r, \dot{r}, \cdots, r^{(i-1)}\right\}$ (for $i \in \mathbb{N}_{2: n+1}$ ). Finally define a $\mathcal{C}^{0}$ function as $\operatorname{sig}^{\alpha}(\cdot) \triangleq|\cdot|^{\alpha} \operatorname{sgn}(\cdot)$ with $\operatorname{sgn}(\cdot)$ denoting the standard signum function.

\section{B. Some Useful Lemmas}

The following lemmas are revisited first since they will play a key role in the development of the main results of the paper.

Lemma 1. [25] For any $x \in \mathbb{R}, y \in \mathbb{R}$, when $\ell \geq$ 1 , the following inequalities hold $|x+y|^{\ell} \leq 2^{\ell-1} \mid x^{\ell}+$ $\left.y^{\ell}\left|, \quad(|x|+|y|)^{\frac{1}{\ell}} \leq\right| x\right|^{\frac{1}{\ell}}+|y|^{\frac{1}{\ell}} \leq 2^{\frac{\ell-1}{\ell}}(|x|+|y|)^{\frac{1}{\ell}}$. When $\ell \in \mathbb{R}_{\text {odd }}^{\geq 1}$, one obtains $|x-y|^{\ell} \leq 2^{\ell-1}\left|x^{\ell}-y^{\ell}\right|,\left|x^{\frac{1}{\ell}}-y^{\frac{1}{\ell}}\right| \leq$ $2^{\frac{\ell-1}{\ell}}|x-y|^{\frac{1}{\ell}}$ and $\left|x^{\ell}-y^{\ell}\right| \leq c|x-y|\left((x-y)^{\ell-1}+y^{\ell-1}\right)$ for a constant $c>0$.

Lemma 2. [24] Let $c, d$ be positive constants. Then, for any real-valued function $\gamma(x, y)>0$, the following inequality holds $|x|^{c}|y|^{d} \leq \frac{c}{c+d} \gamma(x, y)|x|^{c+d}+$ $\frac{d}{c+d} \gamma^{-\frac{c}{d}(c+d)}(x, y)|y|^{c+d}$.

Lemma 3. [21] Consider a continuous nonlinear system $\dot{x}=$ $f(x)$. Suppose there exist a $\mathcal{C}^{1}$ positive definite and proper function $V(x)$, and real numbers $\lambda>0$ and $0<\alpha<1$, such that $\dot{V}(x)+\lambda V^{\alpha}(x) \leq 0$. Then the origin of the system is globally finite-time stable. The convergence time, depending on the initial state $x(0)=x_{0}$, satisfies $T\left(x_{0}\right) \leq \frac{V^{1-\alpha}\left(x_{0}\right)}{\lambda(1-\alpha)}$.

\section{Main Results}

We consider a class of nonlinear systems subject to unmatched time-varying disturbances, depicted by

$$
\begin{aligned}
\dot{x}_{i}(t) & =x_{i+1}(t)+f_{i}\left(\vec{x}_{i}(t)\right)+w_{i}(t) \\
\dot{x}_{n}(t) & =u(t)+f_{n}\left(\vec{x}_{n}(t)\right)+w_{n}(t), y(t)=x_{1}(t)
\end{aligned}
$$

for $i \in \mathbb{N}_{1: n-1}$, where $x_{i} \in \mathbb{R}, u \in \mathbb{R}$ and $y \in \mathbb{R}$ are system state, control input and controlled output, respectively. $w_{i}(t), w_{n}(t) \in \mathbb{D} \subset \mathbb{R}$ with $\mathbb{D}$ being a compact set denote the unmatched and matched external disturbances, respectively. We suppose that the state $x_{i}$ and nonlinear function $f_{i}\left(\vec{x}_{i}\right)$ are available for control design in this letter, which is in general a necessary condition for exact tracking control of nonlinear system (1) [13], [14]. The output tracking error is defined as $\epsilon(t)=y(t)-r(t)$ where $r(t)$ is the desired reference signal. The nonlinear system (1) represents a class of lower triangular nonlinear systems subject to disturbances. Many practical systems can be described by (1) directly or via adequate coordinate transformations, see rigid spacecraft, jet engine compression system, active suspension system and biological rhythm system for examples [3], [5], [9]. The external signals as well as the nonlinearities are supposed to satisfy the following assumptions.

Assumption 1. The external disturbances and reference signal satisfy $w_{i}(t) \in \mathcal{C}^{n+1-i}, r(t) \in \mathcal{C}^{n}$. Furthermore, $w_{i}^{(j)}(t) \in$ $\mathcal{L}_{\infty}$ and $r^{(k)}(t) \in \mathcal{L}_{\infty}$ are bounded by $\sup _{t \geq 0}\left|w_{i}^{(j)}(t)\right| \leq$ $\digamma_{i}^{+}, \sup _{t \geq 0}\left|r^{(k)}(t)\right| \leq \digamma^{+}$for $j \in \mathbb{N}_{0: n+1-i}$ and $k \in \mathbb{N}_{0: n}$, where $\digamma_{i}^{+}$and $\digamma^{+}$are some positive constants.

Remark 1. The disturbances satisfying Assumption 1 represent quite a broad class of exogenous signals, which cover the smooth disturbances governed by neutral stable linear/nonlinear exosystems [5], [9] as a special case. The disturbances handled by the higher-order sliding mode observer [12] should also satisfy this assumption. Actually, any unknown smooth time-varying disturbance satisfies the above assumption.

Assumption 2. There exist a constant $\tau$ (a ratio of an even and an odd integer, which is called homogeneous degree) and a $\mathcal{C}^{0}$ function $\rho_{i}\left(\vec{x}_{i}, \vec{y}_{i}\right)>0$ such that for all $\vec{x}_{i} \in \mathbb{R}^{i}$ and $\vec{y}_{i} \in \mathbb{R}^{i}$, the following inequality holds

$$
\left|f_{i}\left(\vec{x}_{i}\right)-f_{i}\left(\vec{y}_{i}\right)\right| \leq \rho_{i}\left(\vec{x}_{i}, \vec{y}_{i}\right) \sum_{j=1}^{i}\left|x_{j}-y_{j}\right|^{\frac{r_{i}+\tau}{r_{j}}}
$$

for $i \in \mathbb{N}_{1: n}$ with $r_{1}=1$ and $r_{i}+\tau=r_{i+1} \in \mathbb{Q}_{\text {odd }}^{+}$.

Remark 2. Assumption 2 specifies the homogeneous growth condition [25], which covers a large class of nonlinear functions. Taking any $\mathcal{C}^{1}$ nonlinear function $f_{i}\left(\vec{x}_{i}\right)$ as an example, it can be shown that there exist a homogeneous degree $-1<\tau \leq 0$ and a $\mathcal{C}^{0}$ function $\rho_{i}\left(\vec{x}_{i}, \vec{y}_{i}\right)>0$ such that the inequality (2) is satisfied. In addition, for certain $\mathcal{C}^{0}$ but nonsmooth nonlinearities, the inequality (2) is also satisfied by choosing an appropriate $\tau$. For example, by Lemma 1 , the nonlinearity $f_{1}\left(x_{1}\right)=x_{1}^{1 / 3}$ satisfies (2) with $\tau=-2 / 3$ and $\rho_{1}>2^{2 / 3}$. In a conclusion, the condition in 
Assumption 2 is largely relaxed as compared with nonlinear output regulation and traditional backstepping based design since the smoothness of nonlinearities is usually required therein [3], [5].

Control Objective: In this paper, we attempt to address the finite-time exact tracking and disturbance rejection problem of a class of lower triangular nonlinear system (1). We will show that under Assumptions 1 and 2, there exist two functions $M(\mathbf{z}, \mathbf{x}, u): \mathbb{R}^{q} \times \mathbb{R}^{n} \times \mathbb{R} \rightarrow \mathbb{R}^{q}, N(\mathbf{z}, \mathbf{x}, \mathbf{r}): \mathbb{R}^{q} \times \mathbb{R}^{n} \times \mathbb{R}^{n} \rightarrow$ $\mathbb{R}$ and a nonlinear dynamic controller $\dot{\mathbf{z}}=M(\mathbf{z}, \mathbf{x}, u), u=$ $N(\mathbf{z}, \mathbf{x}, \mathbf{r}), \mathbf{z} \in \mathbb{R}^{q}$ with $\mathbf{x}=\vec{x}_{n}$ and $\mathbf{r}=\mathbf{r}_{n}$ ensuring the finite-time exact tracking control of nonlinear system (1) in the sense that all the variables of the closed-loop system satisfy $x \in \mathcal{L}_{\infty}, z \in \mathcal{L}_{\infty}$ and $u \in \mathcal{L}_{\infty}$; and there exists a finite time $T_{g}$ such that $y(t)=r(t)$ for $t \geq T_{g}$.

\section{A. Estimations of Steady States}

Suppose that the disturbance $w_{i}(t)$ in (1) satisfies Assumption 1. A set of disturbance observers are firstly utilized to estimate the disturbances and their derivatives in system (1), given by

$$
\dot{z}_{0}^{i}=v_{0}^{i}+x_{i+1}+f_{i}\left(\vec{x}_{i}\right), \dot{z}_{1}^{i}=v_{1}^{i}, \cdots, \dot{z}_{k}^{i}=v_{k}^{i},
$$

for $i \in \mathbb{N}_{1: n}, \quad k \in \mathbb{N}_{1: n+1-i}$, with definitions $x_{i+1}=u, v_{0}^{i}=$ $z_{1}^{i}-\lambda_{0}^{i} L_{i}^{\alpha_{0}^{i}} \operatorname{sig}^{1-\alpha_{0}^{i}}\left(z_{0}^{i}-x_{i}\right), v_{j}^{i}=z_{j+1}^{i}-\lambda_{j}^{i} L_{i}^{\alpha_{j}^{i}} \operatorname{sig}^{1-\alpha_{j}^{i}}\left(z_{j}^{i}-\right.$ $\left.v_{j-1}^{i}\right)$ and $v_{n+1-i}^{i}=-\lambda_{n+1-i}^{i} L_{i}^{\alpha_{n+1-i}^{i}} \operatorname{sig}^{1-\alpha_{n+1-i}^{i}}\left(z_{n+1-i}^{i}-\right.$ $v_{n-i}^{i}$ ) for $i \in \mathbb{N}_{1: n}, j \in \mathbb{N}_{1: n-i}$, where $\alpha_{j}^{i}=\frac{1}{n+2-i-j}$. Parameters $\lambda_{j}^{i}>0$ and $L_{i}>0$ are observer gains to be assigned. The estimates of state $x_{i}$ and disturbances $w_{i}^{(k-1)}$ are denoted by

$$
\hat{x}_{i}=z_{0}^{i}, \widehat{w_{i}^{(k-1)}}=z_{k}^{i}
$$

for $i \in \mathbb{N}_{1: n}$ and $k \in \mathbb{N}_{1: n+1-i}$. Combining (1) with (3) and (4), the observer estimation error is governed by

$$
\begin{aligned}
\dot{e}_{0}^{i}= & e_{1}^{i}-\lambda_{0}^{i} L_{i}^{\alpha_{0}^{i}} \operatorname{sig}^{1-\alpha_{0}^{i}}\left(e_{0}^{i}\right) \\
\dot{e}_{j}^{i}= & e_{j+1}^{i}-\lambda_{j}^{i} L_{i}^{\alpha_{j}^{i}} \operatorname{sig}^{1-\alpha_{j}^{i}}\left(e_{j}^{i}-\dot{e}_{j-1}^{i}\right) \\
\dot{e}_{n+1-i}^{i} \in & {\left[-\digamma_{i}^{+}, \digamma_{i}^{+}\right]-\lambda_{n+1-i}^{i} L_{i}^{\alpha_{n+1-i}^{i}} } \\
& \times \operatorname{sig}^{1-\alpha_{n+1-i}^{i}}\left(e_{n+1-i}^{i}-\dot{e}_{n-i}^{i}\right)
\end{aligned}
$$

where the estimation errors are defined as $e_{0}^{i}=\hat{x}_{i}-x_{i}, e_{k}^{i}=$ $\widehat{w_{i}^{(k-1)}}-w_{i}^{(k-1)}$ for $k \in \mathbb{N}_{1: n+1-i}$.

Lemma 4. [11], [12] If the observer gain $L_{i}$ is selected such that $L_{i}>\digamma_{i}^{+}$, then the following conditions hold: 1) all the signals $e_{j}^{i}(t)$ for $j \in \mathbb{N}_{0: n+1-i}$ and $z_{k}^{i}(t)$ for $k \in \mathbb{N}_{1: n+1-i}$ are uniformly globally bounded; and 2) the observer error dynamics (5) are finite-time stable, i.e., there exists a time instant $T_{f}$ such that $e_{j}^{i}(t)=0$ for $t \geq T_{f}$.

For the sake of space, the readers are referred to Remark 6.1 in [11] (see page 229) for detailed guidelines on selection of observer parameters $\lambda_{j}^{i}$. The convergence rate of the observer can be adjusted by tuning the observer parameters $L_{i}$.
The steady states of system (1) can be represented by $\pi_{1}(t)=\phi_{1}\left(\mathbf{r}_{1}(t)\right), \pi_{i}(t)=\phi_{i}\left(\mathbf{w}_{i}(t), \mathbf{r}_{i}(t)\right)$ for $i \in \mathbb{N}_{2: n}$, and $\pi_{u}(t)=\phi_{u}\left(\mathbf{w}_{n+1}(t), \mathbf{r}_{n+1}(t)\right)$ with $\pi_{i}(t)$ and $\pi_{u}(t)$ determined by the following equations $\pi_{1}(t)=r(t), \pi_{i}(t)=$ $\frac{d \pi_{i-1}}{d t}(t)-f_{i-1}\left(\vec{\pi}_{i-1}(t)\right)-w_{i-1}(t), \pi_{u}(t)=\frac{d \pi_{n}}{d t}(t)-$ $f_{n}\left(\vec{\pi}_{n}(t)\right)-w_{n}(t)$. Clearly, $\phi_{1}(\cdot), \phi_{i}(\cdot)$ and $\phi_{u}(\cdot)$ are functions in terms of references, disturbances and their various order of derivatives. Replacing the disturbances and their derivatives by their estimates, we obtain the following set of steady-state estimations of system (1)

$$
\begin{aligned}
& \hat{\pi}_{1}(t):=\phi_{1}\left(\mathbf{r}_{1}(t)\right), \hat{\pi}_{i}(t):=\phi_{i}\left(\mathbf{z}_{i}(t), \mathbf{r}_{i}(t)\right) \\
& \hat{\pi}_{u}(t):=\phi_{u}\left(\mathbf{z}_{n+1}(t), \mathbf{r}_{n+1}(t)\right)
\end{aligned}
$$

for $i \in \mathbb{N}_{2: n}$, where $\mathbf{z}_{i}$ (the estimate of $\mathbf{w}_{i}$ ) is a vector in terms of the estimates of references, disturbances and their derivatives generated by disturbance observers (3), defined as $\mathbf{z}_{i}:=\left\{\bar{z}_{i-1,0}, \bar{z}_{i-2,1}, \cdots, \bar{z}_{1, i-2}\right\}$ with $\bar{z}_{i, j}:=$ $\left\{z_{j+1}^{1}, z_{j+1,2}^{2}, \cdots, z_{j+1}^{i-2}\right\}$ for $i \in \mathbb{N}_{2: n+1}$ and $j \in \mathbb{N}_{0: i-2}$. Suppose that $\phi_{i}(\cdot) \in \mathcal{C}^{1}$ for $i \in \mathbb{N}_{1: n}$ and $\phi_{u}(\cdot) \in \mathcal{C}^{0}$ with respect to their respective arguments $\left(\mathbf{z}_{i}(t), \mathbf{r}_{i}(t)\right)$ and $\left(\mathbf{z}_{n+1}(t), \mathbf{r}_{n+1}(t)\right)$ for all $t \geq 0$. The property of finite-time estimation of the steady states is presented by the following lemma.

Lemma 5. The disturbance observers (3) and (4) guarantee that the steady-state estimations (6) of system (1) converge to their real steady-state values in a finite time $T_{f}$, that is, $\hat{\pi}_{i}(t)=\pi_{i}(t), \hat{\pi}_{u}(t)=\pi_{u}(t)$ for $t \geq T_{f}$.

Proof. It can be concluded from the boundedness of disturbances/references (see Assumption 1) that $\mathbf{w}_{i}(t) \in \mathcal{L}_{\infty}$ and $\mathbf{r}_{i}(t) \in \mathcal{L}_{\infty}$. Similarly, it follows from Lemma 4 that $\mathbf{z}_{i}(t) \in \mathcal{L}_{\infty}$. With those derivations in mind, it follows from the conditions $\phi_{i}(\cdot) \in \mathcal{C}^{1}$ and $\phi_{u}(\cdot) \in \mathcal{C}^{0}$ that $\pi_{i}(t) \in \mathcal{L}_{\infty}$, $\dot{\pi}_{i}(t) \in \mathcal{L}_{\infty}, \pi_{u}(t) \in \mathcal{L}_{\infty}, \hat{\pi}_{i}(t) \in \mathcal{L}_{\infty}, \dot{\hat{\pi}}_{i}(t) \in \mathcal{L}_{\infty}$ and $\hat{\pi}_{u}(t) \in \mathcal{L}_{\infty}$. Moreover, since the convergence of disturbance observers reveals that $z_{k}^{i}=w_{i}^{(k-1)}(t)$ (that is $\mathbf{z}_{i}(t)=\mathbf{w}_{i}(t)$ ) for $t \geq T_{f}$, we can draw the conclusion that $\hat{\pi}_{i}(t)=\pi_{i}(t)$ and $\hat{\pi}_{u}(t)=\pi_{u}(t)$ for $t \geq T_{f}$.

\section{B. Controller Design}

For the disturbance estimations $\overrightarrow{\hat{\pi}}_{i}(t)$ generated by finitetime disturbance observers (3) and (4), if the nonlinearities in (1) satisfy Assumption 2, then there exists a $\mathcal{C}^{0}$ function $\gamma_{i}\left(\vec{\eta}_{i}\right) \geq 0$ such that for all $\vec{x}_{i} \in \mathbb{R}^{i}$, the following inequality holds

$$
\left|f_{i}\left(\vec{x}_{i}\right)-f_{i}\left(\overrightarrow{\hat{\pi}}_{i}\right)\right| \leq \gamma_{i}\left(\vec{\eta}_{i}\right) \sum_{j=1}^{i}\left|\eta_{j}\right|^{\frac{r_{i}+\tau}{r_{j}}}, i \in \mathbb{N}_{1: n}
$$

where $\gamma_{i}\left(\vec{\eta}_{i}\right) \geq \rho_{i}\left(\vec{\eta}_{i}+\overrightarrow{\hat{\pi}}_{i}, \overrightarrow{\hat{\pi}}_{i}\right)$ for all $\overrightarrow{\hat{\pi}}_{i} \in \hat{\mathbb{D}}$ with $\hat{\mathbb{D}}$ being a compact set.

Let $\sigma, \rho \in \mathbb{Q}_{\text {odd }}^{+}$satisfying $\sigma \geq \max _{i \in \mathbb{N}_{1: n}}\left\{r_{i}\right\}$ and $\rho \geq \max _{i \in \mathbb{N}_{1: n}}\left\{r_{i}+\tau, \sigma\right\}$. The following virtual dynamic compensators are designed

$\eta_{i}=x_{i}-\phi_{i}\left(\mathbf{z}_{i}, \mathbf{r}_{i}\right), \eta_{i}^{*}=-k_{i-1}\left(\vec{\eta}_{i-1}\right) \xi_{i-1}^{\frac{r_{i}}{\sigma}}, \xi_{i}=\eta_{i}^{\frac{\sigma}{r_{i}}}-\eta_{i}^{* \frac{\sigma}{r_{i}}}$ 
for $i \in \mathbb{N}_{1: n}$, where $\mathbf{z}_{i}$ is dynamically determined by the disturbance observer (3). Following by the transformation (8), the finite-time exact tracking control law is constructed as

$$
u=-\left(\sum_{i=1}^{n} g_{i}\left(\vec{\eta}_{n}\right) \eta_{i}^{\frac{\sigma}{r_{i}}}\right)^{\frac{r_{n+1}}{\sigma}}+\phi_{u}\left(\mathbf{z}_{n+1}, \mathbf{r}_{n+1}\right)
$$

with $g_{i}\left(\vec{\eta}_{n}\right)=\prod_{l=i}^{n} k_{l}^{\frac{\sigma}{r_{l+1}}}\left(\vec{\eta}_{l}\right)$. Denoting $\mathbf{z} \quad:=$ $\left[\mathbf{z}_{1}, \cdots, \mathbf{z}_{n+1}\right]^{T}$, the constructions of $M(\mathbf{z}, \mathbf{x}, u)$ and $N(\mathbf{z}, \mathbf{x}, \mathbf{r})$ are described by disturbance observers (3) and control law (8) with definitions in (9), respectively.

Clearly, to give the detailed expression of the control law, the control gains $g_{i}\left(\vec{\eta}_{n}\right)$ should be determined. To begin with, design a candidate Lyapunov function $V_{1}\left(\vec{\eta}_{1}\right)=$ $\int_{\eta_{1}^{*}}^{\eta_{1}}\left(s^{\frac{\sigma}{r_{1}}}-\eta_{1}^{* \frac{\sigma}{r_{1}}}\right)^{\frac{2 \rho-\tau-r_{1}}{\sigma}} d s$ with virtual control $\eta_{1}^{*}$ designed as $\eta_{1}^{*}=0$. With (6) in mind, the time derivative of $\eta_{1}$ along system dynamics (1) is given by

$$
\dot{\eta}_{1}=\eta_{2}+f_{1}\left(\vec{x}_{1}\right)-f_{1}\left(\overrightarrow{\hat{\pi}}_{1}\right)+\varepsilon_{1}(t)
$$

where $\varepsilon_{1}(t)=f_{1}\left(\overrightarrow{\hat{\pi}}_{1}\right)-f_{1}\left(\vec{\pi}_{1}\right)+\hat{\pi}_{2}-\pi_{2}$. By Lemma 2 , the time derivative of $V_{1}\left(\vec{\eta}_{1}\right)$ along (7) and (10) is

$\dot{V}_{1}\left(\vec{\eta}_{1}\right) \leq \eta_{1}^{\frac{2 \rho-\tau-r_{1}}{r_{1}}}\left(\eta_{2}-\eta_{2}^{*}+\eta_{2}^{*}\right)+\left(\gamma_{1}\left(\eta_{1}\right)+\varrho_{1}\right) \eta_{1}^{\frac{2 \rho}{r_{1}}}+\frac{\varepsilon_{1}^{\frac{2 \rho}{r_{1}+\tau}}}{\nu_{1}}$

where $\nu_{1}=n$ and $\varrho_{1}>0$ is a constant. In the virtual control law $\eta_{2}^{*}$ as shown in (8), we select the control gain $k_{1}\left(\eta_{1}\right)$ such that $k_{1}\left(\eta_{1}\right) \geq \nu_{1}+\gamma_{1}\left(\eta_{1}\right)+\varrho_{1}$. Substituting the virtual control law $\eta_{2}^{*}(8)$ into (11) gives

$$
\dot{V}_{1}\left(\vec{\eta}_{1}\right) \leq \xi_{1}^{\frac{2 \rho-\tau-r_{1}}{\sigma}}\left(\eta_{2}-\eta_{2}^{*}\right)-\nu_{1} \xi_{1}^{\frac{2 \rho}{\sigma}}+\frac{1}{\nu_{1}} \varepsilon_{1}^{\frac{2 \rho}{r_{1}+\tau}}
$$

Lemma 6. Define a $\mathcal{C}^{1}$ Lyapunov function $V_{i}\left(\vec{\eta}_{i}\right)=$ $\sum_{k=1}^{i} \int_{\eta_{i}^{*}}^{\eta_{i}}\left(s^{\frac{\sigma}{r_{i}}}-\eta_{i}^{*} \frac{\sigma}{r_{i}}\right)^{\frac{2 \rho-\tau-r_{i}}{\sigma}} d s$. Suppose that Assumptions 1 and 2 are satisfied. Under the virtual dynamic compensators defined in (8), the derivative of $V_{i}\left(\vec{\eta}_{i}\right)$ along the system (1) satisfies

$\dot{V}_{i}\left(\vec{\eta}_{i}\right) \leq \xi_{i}^{\frac{2 \rho-\tau-r_{i}}{\sigma}}\left(\eta_{i+1}-\eta_{i+1}^{*}\right)-\nu_{i} \sum_{j=1}^{i} \xi_{j}^{\frac{2 \rho}{\sigma}}+\frac{1}{\nu_{i}} \sum_{j=1}^{i} \varepsilon_{j}^{\frac{2 \rho}{r_{j}+\tau}}$

with $\nu_{i}=n+1-i$.

Proof. The proof of the lemma is proceeded in an inductive manner. Suppose at step $i-1$, there exist a $\mathcal{C}^{1}$ Lyapunov function $V_{i-1}\left(\vec{\eta}_{i-1}\right): \mathbb{R}^{i-1} \rightarrow \mathbb{R}$, which is positive definite and proper, and a set of $\mathcal{C}^{0}$ virtual controllers $\eta_{1}^{*}=0$ and $\eta_{2}^{*}, \cdots, \eta_{i}^{*}$ defined by (8) with $\mathcal{C}^{1}$ control gains $k_{j}\left(\vec{\eta}_{j}\right)>0$ such that

$$
\begin{aligned}
\dot{V}_{i-1}\left(\vec{\eta}_{i-1}\right) \leq & \xi_{i-1}^{\frac{2 \rho-\tau-r_{i-1}}{\sigma}}\left(\eta_{i}-\eta_{i}^{*}\right) \\
& -\nu_{i-1} \sum_{j=1}^{i-1} \xi_{j}^{\frac{2 \rho}{\sigma}}+\frac{1}{\nu_{i-1}} \sum_{j=1}^{i-1} \varepsilon_{j}^{\frac{2 \rho}{r_{j}+\tau}}
\end{aligned}
$$

It is obvious that (13) reduces to the inequality (12) when $i=2$ under the definitions of (8). We will show that inequality (13) also holds at step $i$.
With the help of (6), the time derivative of $\eta_{i}$ along system dynamics (1) is governed by

$$
\dot{\eta}_{i}=\eta_{i+1}+f_{i}\left(\vec{x}_{i}\right)-f_{i}\left(\overrightarrow{\hat{\pi}}_{i}\right)+\varepsilon_{i}(t)
$$

where $\varepsilon_{i}(t)=f_{i}\left(\overrightarrow{\hat{\pi}}_{i}\right)-f_{i}\left(\vec{\pi}_{i}\right)+\hat{\pi}_{i+1}-\pi_{i+1}+\dot{\pi}_{i}-\dot{\hat{\pi}}_{i}$. Note that $V_{i}\left(\vec{\eta}_{i}\right)=V_{i-1}\left(\vec{\eta}_{i-1}\right)+W_{i}\left(\vec{\eta}_{i}\right)$ where $W_{i}\left(\vec{\eta}_{i}\right)=$ $\int_{\eta_{i}^{*}}^{\eta_{i}}\left(s^{\frac{\sigma}{r_{i}}}-\eta_{i}^{* \frac{\sigma}{r_{i}}}\right)^{\frac{2 \rho-\tau-r_{i}}{\sigma}} d s$. Consequently, the derivative of $V_{i}\left(\vec{\eta}_{i}\right)$ along (14) is

$$
\begin{aligned}
\dot{V}_{i}\left(\vec{\eta}_{i}\right) \leq & \dot{V}_{i-1}\left(\vec{\eta}_{i-1}\right)+\xi_{i}^{\frac{2 \rho-\tau-r_{i}}{\sigma}}\left(\eta_{i+1}-\eta_{i+1}^{*}\right)+\sum_{l=1}^{i-1} \frac{\partial W_{i}}{\partial \eta_{l}} \dot{\eta}_{l} \\
& +\xi_{i}^{\frac{2 \rho-\tau-r_{i}}{\sigma}}\left(\eta_{i+1}^{*}+f_{i}\left(\vec{x}_{i}\right)-f_{i}\left(\overrightarrow{\hat{\pi}}_{i}\right)+\varepsilon_{i}(t)\right)
\end{aligned}
$$

for a virtual control $\eta_{i+1}^{*}$ to be designed later. Each terms in the right hand side of (15) are estimated as follows.

By Lemmas 1 and 2 we have ${ }^{1}$

$$
\left|\xi_{i-1}^{\frac{2 \rho-\tau-r_{i-1}}{\sigma}}\left(\eta_{i}-\eta_{i}^{*}\right)\right| \leq \frac{1}{3} \xi_{i-1}^{\frac{2 \rho}{\sigma}}+\bar{c}_{i} \xi_{i}^{\frac{2 \rho}{\sigma}}
$$

Define $\kappa_{l}\left(\vec{\eta}_{l}\right)=k_{l}^{\frac{\sigma}{r_{l+1}}}\left(\vec{\eta}_{l}\right)$ for $l \in \mathbb{N}_{1: i-1}$. By Lemma 1 and Assumption 2, we have

$$
\begin{aligned}
& \left|\xi_{i}^{\frac{2 \rho-\tau-r_{i}}{\sigma}}\left(f_{i}\left(\vec{x}_{i}\right)-f_{i}\left(\overrightarrow{\hat{\pi}}_{i}\right)+\varepsilon_{i}\right)\right| \\
& \leq\left|\xi_{i}^{\frac{2 \rho-r_{i+1}}{\sigma}}\right|\left(\gamma_{i}\left(\vec{\eta}_{i}\right) \sum_{j=1}^{i}\left|\xi_{j}-\kappa_{j-1} \xi_{j-1}\right|^{\frac{r_{i+1}}{\sigma}}+\left|\varepsilon_{i}\right|\right) \\
& \leq \frac{1}{2} \sum_{j=1}^{i-2} \xi_{j}^{\frac{2 \rho}{\sigma}}+\frac{1}{3} \xi_{i-1}^{\frac{2 \rho}{\sigma}}+\frac{1}{\nu_{i}} \varepsilon_{i}^{\frac{2 \rho}{r_{i+1}}}+\hat{c}_{i}\left(\vec{\eta}_{i}\right) \xi_{i}^{\frac{2 \rho}{\sigma}}
\end{aligned}
$$

Using Lemmas 1 and 2, after tedious calculations, it follows from Assumptions 1 and 2 that

$$
\begin{aligned}
\sum_{l=1}^{i-1}\left|\frac{\partial W_{i}}{\partial \eta_{l}} \dot{\eta}_{l}\right| \leq & \frac{1}{2} \sum_{j=1}^{i-2} \xi_{j}^{\frac{2 \rho}{\sigma}}+\frac{1}{3} \xi_{i-1}^{\frac{2 \rho}{\sigma}}+\tilde{c}_{i-1}\left(\vec{\eta}_{i-1}\right) \xi_{i}^{\frac{2 \rho}{\sigma}} \\
& +\frac{1}{\nu_{i} \nu_{i-1}} \sum_{j=1}^{i-1} \varepsilon_{j}^{\frac{2 \rho}{r_{j+1}}}
\end{aligned}
$$

The proof of (18) is provided in Appendix for readability. Substituting (13) and estimations (16)-(18) into (15) gives

$$
\begin{aligned}
\dot{V}_{i}\left(\vec{\eta}_{i}\right) \leq & \xi_{i}^{\frac{2 \rho-\tau-r_{i}}{\sigma}}\left(\eta_{i+1}-\eta_{i+1}^{*}\right)-\nu_{i} \sum_{j=1}^{i-1} \xi_{j}^{\frac{2 \rho}{\sigma}} \\
& +\check{c}_{i}\left(\vec{\eta}_{i}\right) \xi_{i}^{\frac{2 \rho}{\sigma}}+\frac{1}{\nu_{i}} \sum_{j=1}^{i} \varepsilon_{j}^{\frac{2 \rho}{r_{j+1}}}+\xi_{i}^{\frac{2 \rho-\tau-r_{i}}{\sigma}} \eta_{i+1}^{*}
\end{aligned}
$$

where $\left.\check{c}_{i}\left(\vec{\eta}_{i}\right)=\vec{\eta}_{i-1}\right)+\hat{c}_{i}\left(\vec{\eta}_{i}\right)+\bar{c}_{i}$. Substituting a virtual control law $\eta_{i+1}^{*}=-k_{i}\left(\vec{\eta}_{i}\right) \xi_{i}^{\frac{r_{i+1}}{\sigma}}$ (8) with $k_{i}\left(\vec{\eta}_{i}\right) \geq \nu_{i}+\check{c}_{i}\left(\vec{\eta}_{i}\right)$ into (19) yields the conclusion. This completes the proof.

\footnotetext{
${ }^{1}$ For briefness of expressions, we use the symbols $\bar{c}_{i}, \hat{c}_{i}(\cdot)$ and $\tilde{c}_{i-1}(\cdot)$ denoting some constants or functions in terms of $\vec{\eta}_{i}$ in the following.
} 


\section{Performance Analysis}

To begin with, we present the following lemma.

Lemma 7. If $f_{i} \in \mathcal{C}^{0}$, the disturbance observers (3) and (4) ensure that the error signal $\varepsilon_{i}(t)$ governed by (10) and (14) satisfies $\varepsilon_{i}(t) \in \mathcal{L}_{\infty}$ and $\varepsilon_{i}(t)=0$ for $t \geq T_{f}$.

Proof. Since $f_{i} \in \mathcal{C}^{0}$, by Lemma 5 , it directly follows from (10) and (14) that $\varepsilon_{i}(t) \in \mathcal{L}_{\infty}$ and $\varepsilon_{i}(t)=0$ for $t \geq T_{f}$.

The performances of the closed-loop system are concluded by the following theorem.

Theorem 1. Under Assumptions 1 and 2, the proposed composite controller consisting of (3), (4), (6) and (9) guarantees the stability and the asymptotical exact tracking control performance of system (1). Furthermore, the finite-time exact tracking performance of the proposed method is guaranteed by determining a homogenous degree $\tau$ satisfying $-1<\tau<0$.

Proof. Using the definition of (6), the time derivative of $\eta_{n}$ along system dynamics (1) is

$$
\dot{\eta}_{n}=u-\hat{\pi}_{u}+f_{n}\left(\vec{x}_{n}\right)-f_{n}\left(\overrightarrow{\hat{\pi}}_{n}\right)+\varepsilon_{n}(t)
$$

where $\varepsilon_{n}(t)=f_{n}\left(\overrightarrow{\hat{\pi}}_{n}\right)-f_{i}\left(\vec{\pi}_{n}\right)+\hat{\pi}_{u}-\pi_{u}+\dot{\pi}_{n}-\dot{\hat{\pi}}_{n}$. Finally substituting the control law (9) into (20), it follows from (19)

$$
\dot{V}_{n}\left(\vec{\eta}_{n}\right) \leq-\sum_{j=1}^{n} \xi_{j}^{\frac{2 \rho}{\sigma}}+\sum_{j=1}^{n} \varepsilon_{j}^{\frac{2 \rho}{r_{j+1}}}
$$

where the control gain $k_{n}\left(\vec{\eta}_{n}\right)$ is selected such that $k_{n}\left(\vec{\eta}_{n}\right) \geq$ $\nu_{n}+\tilde{c}_{n-1}\left(\vec{\eta}_{n-1}\right)+\hat{c}_{n}\left(\vec{\eta}_{n}\right)+\bar{c}_{n}$. The sketch of the rest proof is divided into two steps as follows.

Step 1 (Boundedness of Closed-Loop System States): By Lemma 7 , the conclusion $\varepsilon_{i} \in \mathcal{L}_{\infty}$ indicates that there exists a constant $c>0$ such that $c=\sup _{t \geq 0}\left(\sum_{j=1}^{n} \varepsilon_{j}^{\frac{2 \rho}{r_{j+1}}}(t)\right)$. Consequently, it can be derived from (21) that $\xi_{i} \in \mathcal{L}_{\infty}$, $\eta_{i} \in \mathcal{L}_{\infty}$ and $x_{i} \in \mathcal{L}_{\infty}$ with the ultimate bound defined by $\Omega_{\eta}=\left\{\left(\eta_{1}, \eta_{2}, \cdots, \eta_{n}\right) \mid \sum_{j=1}^{n} \xi_{j}^{\frac{2 \rho}{\sigma}} \leq c\right\}$. This implies that the closed-loop system will not escape to infinity during the transient of the observers.

Step 2 (Exact Tracking Performance): Since $\varepsilon_{i} \in \mathcal{L}_{\infty}, \eta_{i} \in$ $\mathcal{L}_{\infty}$ and $\varepsilon_{i}(t)=0$ for $t \geq T_{f}$, we have

$$
\dot{V}_{n}\left(\vec{\eta}_{n}\right) \leq-\sum_{j=1}^{n} \xi_{j}^{\frac{2 \rho}{\sigma}}
$$

for all $t \geq T_{f}$. Straightforwardly utilizing LaSalle invariant principal reveals that $\xi_{i}(t) \rightarrow 0, \eta_{i}(t) \rightarrow 0$ and hence $x_{i}(t) \rightarrow \pi_{i}(t)$ as $t \rightarrow \infty$, which shows the asymptotically exact tracking performance of the closed-loop system. Furthermore, by definition of $V_{n}$ and Lemma 1 , we have the following inequality

$$
V_{n}\left(\vec{\eta}_{n}\right) \leq \mu \sum_{j=1}^{n}\left|\xi_{j}\right|^{\frac{2 \rho-\tau}{\sigma}}
$$

where $\mu$ is a positive constant. Let $\lambda=\frac{\mu^{(2 \rho-\tau) / 2 \rho}}{2}$. Using Lemma 1, it can be concluded from (22) and (23) that

$$
\dot{V}_{n}\left(\vec{\eta}_{n}\right)+\lambda V_{n}^{\frac{2 \rho}{2 \rho-\tau}}\left(\vec{\eta}_{n}\right) \leq-\frac{1}{2} \sum_{j=1}^{n}\left|\xi_{j}\right|^{\frac{2 \rho}{\sigma}}
$$
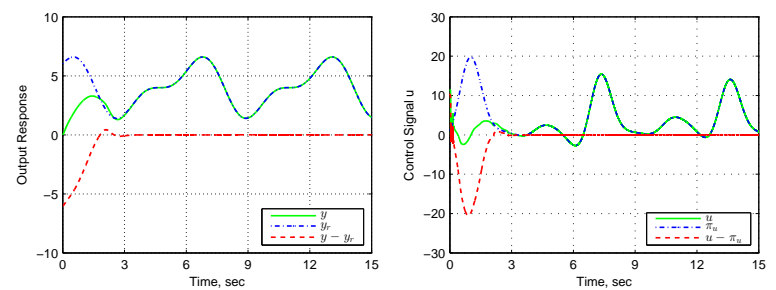

Fig. 1. Response curves of system (25) under the proposed control law.

for $t \geq T_{f}$. Since $-1<\tau<0$, using Lemma 3 , it follows from (24) that there exists a finite time $T_{g}>T_{f}$ such that $\xi_{i}(t)=0, \eta_{i}(t)=0$ and subsequently $x_{i}(t)=\pi_{i}(t)$ for $t \geq T_{g}$.

This completes the proof.

\section{Simulations}

Consider the following nonlinear dynamic system under disturbances

$$
\begin{aligned}
& \dot{x}_{1}=x_{2}+0.5 x_{1}^{3 / 5}+w_{1}(t), \\
& \dot{x}_{2}=10 u+x_{1}^{2} x_{2}+w_{2}(t) .
\end{aligned}
$$

The reference signal to be tracked is given by $r(t)=$ $4+\sin (2 t)+2 \cos (t)$. The disturbance signals for simulation studies are depicted by $w_{1}(t)=1+\sin (\pi t / 4)$ and $w_{2}(t)=1.5+\cos (\pi t / 2)$. Let $\tau=-2 / 5, r_{1}=1, r_{2}=3 / 5$, $r_{3}=1 / 5$ and $\sigma=\rho=1$. Some brief calculations reveal that $f_{1}\left(x_{1}\right)=0.5 x_{1}^{3 / 5}$ and $f_{2}\left(\vec{x}_{2}\right)=x_{1}^{2} x_{2}$ satisfy (7) with $\gamma_{1}\left(\eta_{1}\right)$ a positive constant and $\gamma_{2}\left(\vec{\eta}_{2}\right)=c\left(c_{1} \eta_{1}^{8 / 3}+c_{2} \eta_{2}^{8 / 3}+1\right)$ a $\mathcal{C}^{0}$ function.

With the design procedure described in the last section, the finite-time exact tracking control law for system (25) is given by $\left.u=-k_{2}\left(\vec{\eta}_{2}\right)\right)\left(\eta_{2}^{\frac{5}{3}}+k_{1}^{\frac{5}{3}} \eta_{1}\right)^{\frac{1}{5}}+\hat{\pi}_{u}$ with $k_{2}\left(\vec{\eta}_{2}\right)=k_{2 c}+$ $k_{2 \gamma_{1}}\left|\eta_{1}\right|^{3}+k_{2 \gamma_{2}}\left|\eta_{2}\right|^{3}$. The control parameters are chosen as $k_{1}=1, k_{2 c}=5, k_{2 \gamma_{1}}=0.3$ and $k_{2 \gamma_{2}}=1$. A 3rd-order and a 2nd-order disturbance observers are utilized to obtain the disturbance information of $w_{1}$ and $w_{2}$, respectively. The observer parameters are selected as $\lambda_{0}=2, \lambda_{1}=1.5, \lambda_{2}=$ 1.1 and $L_{1}=L_{2}=5$.

Simulation results of the output and the control input for system (25) are shown by Fig. 1. The steady-state estimations are given in Fig. 2. It can be observed from Fig. 1 that the output $y$ tracks the reference signal $r$ well under the finite-time control law even in the presence of unknown disturbances. It is also shown that after a short transient period the control input $u$ tends to its pre-calculated steady-state value $\pi_{u}$. The convergence of the steady-state estimations is clearly shown in Fig. 2.

\section{CONCLUSION}

The finite-time exact tracking and disturbance rejection problem has been investigated for nonlinear systems under unmatched time-varying disturbances using feedback domination and finite-time disturbance observation techniques. The unmatched disturbances have been exactly compensated in 

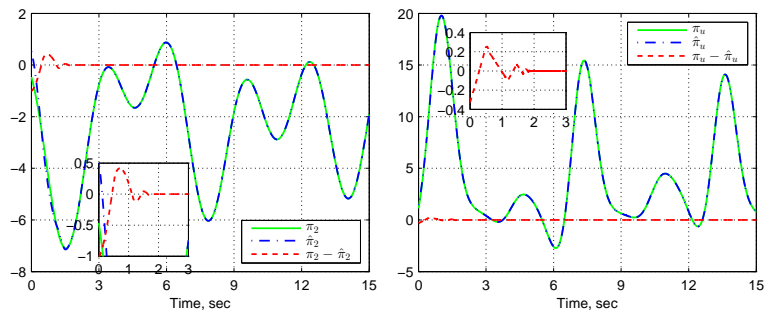

Fig. 2. Response curves of the estimations of the invariant variables.

each step of the virtual control law design. It has been shown that the tracking error of the closed-loop system converges to zero in finite time by the proposed method. Numerical simulation results have shown the performance of the proposed composite controller. Future works will focus on saturation issue of the present control approach.

\section{APPENDIX: PRoOF OF INEQUALITy (18)}

By Lemma 1 and definition of $W_{i}$, for $l \in \mathbb{N}_{1: i-1}$, we have

$$
\left|\frac{\partial W_{i}}{\partial \eta_{l}}\right| \leq \hat{\varrho}_{l}\left|\xi_{i}\right|^{\frac{2 \rho-\tau-\sigma}{\sigma}}\left|\frac{\partial \eta_{i}^{* \frac{\sigma}{r_{i}}}}{\partial \eta_{l}}\right|
$$

By (8), we have $\eta_{i}^{* \frac{\sigma}{r_{i}}}=-\sum_{l=1}^{i-1} \bar{\kappa}_{l}\left(\vec{\eta}_{i-1}\right) \eta_{l}^{\frac{\sigma}{r_{l}}}$ where $\bar{\kappa}_{l}\left(\vec{\eta}_{i-1}\right)=\kappa_{l}\left(\vec{\eta}_{l}\right) \cdots \kappa_{i-2}\left(\vec{\eta}_{i-2}\right) \kappa_{i-1}\left(\vec{\eta}_{i-1}\right)$. Taking derivative of both sides of $\eta_{i}^{* \frac{\sigma}{r_{i}}}$ with respect to $\eta_{l}$ gives

$$
\begin{aligned}
\frac{\partial \eta_{i}^{* \frac{\sigma}{r_{i}}}}{\partial \eta_{l}} & \leq \check{\kappa}_{i-1}\left(\vec{\eta}_{i-1}\right)\left(\sum_{j=1}^{i-1}\left|\xi_{j}\right|+\xi_{l-1}^{\frac{\sigma-r_{l}}{\sigma}}+\xi_{l}^{\frac{\sigma-r_{l}}{\sigma}}\right) \\
& \leq \tilde{\kappa}_{i-1}\left(\vec{\eta}_{i-1}\right) \sum_{j=1}^{i-1} \xi_{j}^{\frac{\sigma-r_{l}}{\sigma}}
\end{aligned}
$$

By Assumption 2, Lemmas 1 and 2, we have

$$
\begin{aligned}
\left|\dot{\eta}_{l}\right| \leq & \left|\xi_{l+1}\right|^{\frac{r_{l+1}}{\sigma}}+k_{l}\left(\vec{\eta}_{l}\right)\left|\xi_{l}\right|^{\frac{r_{l+1}}{\sigma}}+\left|\varepsilon_{l}\right| \\
& +\gamma_{l}\left(\vec{\eta}_{l}\right) \sum_{j=1}^{l}\left(\left|\xi_{j}\right|^{\frac{r_{l+1}}{\sigma}}+\kappa_{j-1}^{\frac{r_{l+1}}{\sigma}}\left|\xi_{j-1}\right|^{\frac{r_{l+1}}{\sigma}}\right) \\
\leq & \tilde{\gamma}_{l}\left(\vec{\eta}_{l}\right) \sum_{j=1}^{l+1}\left|\xi_{j}\right|^{\frac{r_{l+1}}{\sigma}}+\left|\varepsilon_{l}\right|
\end{aligned}
$$

Combining (26), (27) and (28) gives

$$
\begin{aligned}
&\left|\frac{\partial W_{i}}{\partial \eta_{l}} \dot{\eta}_{l}\right| \leq \check{b}_{l}\left(\vec{\eta}_{i-1}\right)\left|\xi_{i}\right|^{\frac{2 \rho-\tau-\sigma}{\sigma}}\left(\sum_{j=1}^{i}\left|\xi_{j}\right|^{\frac{\sigma+\tau}{\sigma}}+\left|\varepsilon_{l}\right|^{\frac{\sigma+\tau}{r_{l+1}}}\right) \\
& \leq \frac{1}{2(i-1)} \sum_{j=1}^{i-2} \xi_{j}^{\frac{2 \rho}{\sigma}}+\frac{1}{3(i-1)} \xi_{i-1}^{\frac{2 \rho}{\sigma}} \\
&+\frac{1}{\nu_{i} \nu_{i-1}} \varepsilon_{l}^{\frac{2 \rho}{r_{l}}}+\tilde{b}_{l}\left(\vec{\eta}_{i-1}\right) \xi_{i}^{\frac{2 \rho}{\sigma}}
\end{aligned}
$$

The inequality (18) is directly obtained by taking the sum of (29) in terms of $l$.

\section{REFERENCES}

[1] P. Apkarian, D. Noll, and A. Rondepierre. "Mixed $H_{2} / H_{\infty}$ control via nonsmooth optimization," SIAM J. Control Optim., vol. 47, no. 3, pp. 1516-1546, 2008.

[2] J. Doyle, K. Glover, P. Khargonekar, and B. Francis, "State-space solutions to standard $\mathrm{H}_{2}$ and $\mathrm{H}_{\infty}$ control problems," IEEE Trans. Autom. Control, vol. 34, no. 2, pp. 831-847, 1989.

[3] M. Krstić, I. Kanellakopoulos, and P. Kokotović, Nonlinear and Adaptive Control Design. New York: Wiley, 1995.

[4] A. Isidori and C. Byrnes, "Output regulation of nonlinear systems," IEEE Trans. Autom. Control, vol. 35, no. 2, pp. 131-140, 1990.

[5] J. Huang, Nonlinear Output Regulation: Theory and Applications. Philadelphia: SIAM Press, 2004.

[6] J. Back, and H. Shim, "An almost necessary and sufficient condition for robust stability of closed-loop systems with disturbance observer," Automatica, vol. 45, no. 1, pp. 296-299, 2008.

[7] X.J. Wei, Z.J. Wu, and H.R. Karimi, "Disturbance observer-based disturbance attenuation control for a class of stochastic systems," Automatica, vol. 63 , pp. $21-25,2016$

[8] J. Han, "From PID to active disturbance rejection control," IEEE Trans. Ind. Electron., vol. 56, no. 3, pp. 900-906, 2009.

[9] Z. Ding, Nonlinear and Adaptive Control Systems. London: IET, 2013.

[10] Z. Chen, and J. Huang, "Global robust output regulation for output feedback systems," IEEE Trans. Autom. Control, vol. 50, no. 1, pp. 117-121, 2005.

[11] Y. Shtessel, C. Edwards, L. Fridman, and A. Levant, Sliding Mode Control and Observation. New York: Birkhäuser, 2014.

[12] A. Levant, "Higher-order sliding modes, differentiation and outputfeedback control," Int. J. Control, vol. 76, no. 9-10, pp. 924-941, 2003.

[13] J. Davila, "Exact tracking using backstepping control design and highorder sliding modes," IEEE Trans. Autom. Control, vol. 58, no. 8, pp. 2077-2081, 2013.

[14] M. Chen, P. Shi, and C.C. Lim, "Robust constrained control for MIMO nonlinear systems based on disturbance observer," IEEE Trans. Autom. Control, vol. 60, no. 12, pp. 3281-3286, 2015.

[15] A. Estrada, L. Fridman, and R. Iriarte, "Combined backstepping and HOSM control design for a class of nonlinear MIMO systems," Int. J. Robust Nonlinear Control, vol. 27, no. 4, pp. 566-581, 2017.

[16] M. Basin, C. Panathula, Y. Shtessel, and P. Rodriguez-Ramirez, "Continuous finite-time output regulators for systems with unmatched disturbances," IEEE Trans. Ind. Electron., vol. 63, no. 8, pp. 5036-5043, 2017.

[17] S. Li, H. Sun, J. Yang, and X. Yu, "Continuous finite-time output regulation for disturbed systems under mismatching condition," IEEE Trans. Autom. Control, vol. 60, no. 1, pp. 277-282, 2015.

[18] A.F. de Loza, J. Cieslak, D. Henry, A. Zolghadri, and L.M. Fridman, "Output tracking of systems subjected to perturbations and a class of actuator faults based on HOSM observation and identification," Automatica, vol. 59, pp. 200-205, 2015.

[19] X. Huang and W. Lin, "Global finite-time stabilization of a class of uncertain nonlinear systems," Automatica, vol. 41, no. 5, pp. 881-888, 2005.

[20] Y. Shen and Y.H. Huang, "Uniformly observable and globally Lipschitzian nonlinear systems admit global finite-time observers," IEEE Trans. Autom. Control, vol. 29, no. 7, pp. 789-801, 2009.

[21] S. P. Bhat and D. S. Bernstein, "Finite-time stability of homogeneous systems," in Proc. Amer. Control Conf., 1997, pp. 2513-2514.

[22] S. Khoo, J. Yin, Z. Man, and X. Yu, "Finite-time stabilization of stochastic nonlinear systems in strict-feedback form," Automatica, vol. 49, pp. 1403-1410, 2013.

[23] Y. Hong and Z.P. Jiang, "Finite-time stabilization of nonlinear systems with parametric and dynamic uncertainties," IEEE Trans. Autom. Control, vol. 51. no. 12, pp. 1950-1956, 2006.

[24] C. Qian and W. Lin, "A continuous feedback approach to global strong stabilization of nonlinear systems," IEEE Trans. Autom. Control, vol. 46, no. 7, pp. 1061-1079, 2001.

[25] J. Polendo and C. Qian, "A generalized homogeneous domination approach for global stabilization of inherently nonlinear systems via output feedback," Int. J. Robust Nonlinear Control, vol. 17, no. 7, pp. 605-629, 2007. 